International Journal of Pure and Applied Mathematics

Volume 86 No. 2 2013, 301-323

ISSN: 1311-8080 (printed version); ISSN: 1314-3395 (on-line version)

url: http://www.ijpam.eu

doi: http://dx.doi.org/10.12732/ijpam.v86i2.6

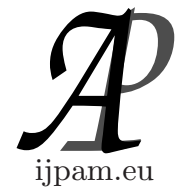

\title{
TIMED PETRI NETS MODELING AND LYAPUNOV/MAX-PLUS ALGEBRA STABILITY ANALYSIS FOR A TYPE OF QUEUING SYSTEMS
}

\author{
Zvi Retchkiman Konigsberg \\ Instituto Politécnico Nacional, CIC \\ Mineria 17-2, Col. Escandon, Mexico D.F 11800, MEXICO
}

\begin{abstract}
A queuing system, is a dynamical system whose state evolves in time by the occurrence of events at possibly irregular time intervals. Placetransitions Petri nets (commonly called Petri nets) are a graphical and mathematical modeling tool applicable to queuing systems in order to represent its states evolution. Timed Petri nets are an extension of Petri nets, where now the timing at which the state changes is taken into consideration. One of the most important performance issues to be considered in a queuing system is its stability. Lyapunov stability theory provides the required tools needed to aboard the stability problem for queuing systems modeled with timed Petri nets whose mathematical model is given in terms of difference equations. By proving practical stability one is allowed to preassigned the bound on the queuing systems dynamics performance. Moreover, employing Lyapunov methods, a sufficient condition for the stabilization problem is also obtained. It is shown that it is possible to restrict the queuing systems state space in such a way that boundedness is guaranteed. However, this restriction results to be vague. This inconvenience is overcome by considering a specific recurrence equation, in the max-plus algebra, which is assigned to the timed Petri net graphical model. In this paper, the modeling and stability problem for a type of queueing systems is addressed. Two classes of queues are considered. The first one (one and two
\end{abstract}

Received: January 15, 2013

(c) 2013 Academic Publications, Ltd. url: www.acadpubl.eu 
servers) working under normal operation, while the second one (one server) having failures in its normal operation due to server breakdown and consumer interruption during service.

AMS Subject Classification: 08A99, 93D35, 93D99, 39A11, 08C99, 16Y60, 65F15, 05C50, 15A29, 15A33

Key Words: queuing systems, Lyapunov methods, max-plus algebra, timed Petri nets

\section{Introduction}

A queuing system, is a dynamical system whose state evolves in time by the occurrence of events at possibly irregular time intervals. Place-transitions Petri nets (commonly called Petri nets) are a graphical and mathematical modeling tool that can be applied to queuing systems in order to represent its states evolution. Petri nets are known to be useful for analyzing the systems properties in addition of being a paradigm for describing and studying information processing systems. Timed Petri nets are an extension of Petri nets, where now the timing at which the state changes is taken into consideration. This is of critical importance since it allows to consider useful measures of performance as for example: how long does the queuing system spends at a given state etc. For a detailed discussion of Petri net theory see [1] and the references quoted therein. One of the most important performance issues to be considered in a queuing system is its stability. Lyapunov stability theory provides the required tools needed to aboard the stability problem for queuing systems modeled with timed Petri nets whose mathematical model is given in terms of difference equations [2]. By proving practical stability one is allowed to preassigned the bound on the queuing systems dynamics performance. Moreover, employing Lyapunov methods, a sufficient condition for the stabilization problem is also obtained. It is shown that it is possible to restrict the queuing systems state space in such a way that boundedness is guaranteed. However, this restriction results to be vague. This inconvenience is overcome by considering a specific recurrence equation, in the max-plus algebra, which is assigned to the the timed Petri net graphical model. This paper proposes a new methodology consisting in combining Lyapunov theory with max-plus algebra to give a precise solution to the stability and scheduling design problem for a type of queuing systems modeled with timed Petri nets. Two classes of queues are considered. The first one (one and two servers) working under normal operation, while the second one (one server) having failures in its normal operation due to server breakdown and 
consumer interruption during service. The presented methodology applied to queuing systems is new and results to be innovative. It is worth mentioning the work done in [3] where the stability for parallel queuing systems is addressed following a stochastic approach. The paper is organized as follows. In Section 2, Lyapunov theory for queuing systems modeled with Petri nets is given. Section 3 , presents max-plus algebra. In Section 4, max-plus recurrence equations for timed event Petri nets are discussed. Section 5, considers the solution to the stability problem for queuing systems modeled with Petri nets. In Section 6, the modeling and stability analysis for the type of queueing systems described above is addressed. Finally, the paper ends with some conclusions.

\section{Lyapunov Stability and Stabilization of Queuing Systems Modeled with Petri Nets}

The solution to the stability problem for queuing systems, whose model is obtained employing timed Petri nets, is achieved thanks to the theory of vector Lyapunov functions and comparison principles. The methodology shows that it is possible to restrict the systems state space in such a way that boundedness is guaranteed.

Notation. $N=\{0,1,2, \ldots\}, R_{+}=[0, \infty), N_{n_{0}}^{+}=\left\{n_{0}, n_{0}+1, \ldots, n_{0}+k, \ldots\right\}$, $n_{0} \geq 0$. Given $x, y \in R^{n}$, we usually denote the relation " $\leq$ " to mean componentwise inequalities with the same relation, i.e., $x \leq y$ is equivalent to $x_{i} \leq y_{i}, \forall i$. A function $f(n, x), f: N_{n_{0}}^{+} \times R^{n} \rightarrow R^{n}$ is called nondecreasing in $x$ if given $x, y \in R^{n}$ such that $x \geq y$ and $n \in N_{n_{0}}^{+}$then, $f(n, x) \geq f(n, y)$.

Consider systems of first ordinary difference equations given by

$$
x(n+1)=f[n, x(n)], x\left(n_{o}\right)=x_{0}, n \in N_{n_{0}}^{+}
$$

where $n \in N_{n_{0}}^{+}, x(n) \in R^{n}$ and $f: N_{n_{0}}^{+} \times R^{n} \rightarrow R^{n}$ is continuous in $x(n)$.

Definition 1. The $n$ vector valued function $\Phi\left(n, n_{0}, x_{0}\right)$ is said to be a solution of (1) if $\Phi\left(n_{0}, n_{0}, x_{0}\right)=x_{0}$ and $\Phi\left(n+1, n_{0}, x_{0}\right)=f\left(n, \Phi\left(n, n_{0}, x_{0}\right)\right)$ for all $n \in N_{n_{0}}^{+}$.

Definition 2. The system (1) is said to be

i) Practically stable, if given $(\lambda, A)$ with $0<\lambda<A$, then

$$
\left|x_{0}\right|<\lambda \Rightarrow\left|x\left(n, n_{0}, x_{0}\right)\right|<A, \forall n \in N_{n_{0}}^{+}, n_{0} \geq 0 ;
$$

ii) Uniformly practically stable, if it is practically stable for every $n_{0} \geq 0$. 
The following class of function is defined.

Definition 3. A continuous function $\alpha:[0, \infty) \rightarrow[0, \infty)$ is said to belong to class $\mathcal{K}$ if $\alpha(0)=0$ and it is strictly increasing.

Consider a vector Lyapunov function $v(n, x(n)), v: N_{n_{0}}^{+} \times R^{n} \rightarrow R_{+}^{p}$ and define the variation of $v$ relative to (1) by

$$
\Delta v=v(n+1, x(n+1))-v(n, x(n))
$$

Then, the following result concerns the practical stability of (1).

Theorem 4. [4] Let $v: N_{n_{0}}^{+} \times R^{n} \rightarrow R_{+}^{p}$ be a continuous function in $x$, define the function $v_{0}(n, x(n))=\sum_{i=1}^{p} v_{i}(n, x(n))$ such that satisfies the estimates

$$
\begin{gathered}
b(|x|) \leq v_{0}(n, x(n)) \leq a(|x|) \text { for } a, b \in \mathcal{K} \text { and } \\
\Delta v(n, x(n)) \leq w(n, v(n, x(n)))
\end{gathered}
$$

for $n \in N_{n_{0}}^{+}, x(n) \in R^{n}$, where $w: N_{n_{0}}^{+} \times R_{+}^{p} \rightarrow R^{p}$ is a continuous function in the second argument.

Assume that: $g(n, e) \triangleq e+w(n, e)$ is nondecreasing in $e, 0<\lambda<A$ are given and finally that $a(\lambda)<b(A)$ is satisfied.

Then, the practical stability properties of

$$
e(n+1)=g(n, e(n)), e\left(n_{0}\right)=e_{0} \geq 0 .
$$

imply the practical stability properties of system (1).

Corollary 5. In Theorem (4):

i) If $w(n, e) \equiv 0$ we get uniform practical stability of (1) which implies structural stability.

ii) If $w(n, e)=-c(e)$, for $c \in \mathcal{K}$, we get uniform practical asymptotic stability of (1).

Definition 6. A Petri net is a 5-tuple, $P N=\left\{P, T, F, W, M_{0}\right\}$ where:

$P=\left\{p_{1}, p_{2}, \ldots, p_{m}\right\}$ is a finite set of places,

$T=\left\{t_{1}, t_{2}, \ldots, t_{n}\right\}$ is a finite set of transitions,

$F \subset(P \times T) \cup(T \times P)$ is a set of arcs,

$W: F \rightarrow N_{1}^{+}$is a weight function,

$M_{0}: P \rightarrow N$ is the initial marking,

$P \cap T=\varnothing$ and $P \cup T \neq \varnothing$. 
Definition 7. The clock structure associated with a place $p_{i} \in P$ is a set $\mathbf{V}=\left\{V_{i}: p_{i} \in P\right\}$ of clock sequences $V_{i}=\left\{v_{i, 1}, v_{i, 2}, \ldots\right\}, v_{i, k} \in R^{+}, k=1,2, \ldots$

The positive number $v_{i, k}$, associated to $p_{i} \in P$, called holding time, represents the time that a token must spend in this place until its outputs enabled transitions $t_{i, 1}, t_{i, 2}, \ldots$, fire. Some places may have a zero holding time while others not. Thus, we partition $P$ into subsets $P_{0}$ and $P_{h}$, where $P_{0}$ is the set of places with zero holding time, and $P_{h}$ is the set of places that have some holding time.

Definition 8. A timed Petri net is a 6-tuple $T P N=\left\{P, T, F, W, M_{0}, \mathbf{V}\right\}$ where $\left\{P, T, F, W, M_{0}\right\}$ are as before, and $\mathbf{V}=\left\{V_{i}: p_{i} \in P\right\}$ is a clock structure. A timed Petri net is a timed event petri net when every $p_{i} \in P$ has one input and one output transition, in which case the associated clock structure set of a place $p_{i} \in P$ reduces to one element $V_{i}=\left\{v_{i}\right\}$

A $P N$ structure without any specific initial marking is denoted by $N$. A Petri net with the given initial marking is denoted by $\left(N, M_{0}\right)$. Notice that if $W(p, t)=\alpha($ or $W(t, p)=\beta)$ then, this is often represented graphically by $\alpha$, $(\beta)$ arcs from $p$ to $t$ ( $t$ to $p$ ) each with no numeric label.

Let $M_{k}\left(p_{i}\right)$ denote the marking (i.e., the number of tokens) at place $p_{i} \in P$ at time $k$ and let $M_{k}=\left[M_{k}\left(p_{1}\right), \ldots, M_{k}\left(p_{m}\right)\right]^{T}$ denote the marking (state) of $P N$ at time $k$. A transition $t_{j} \in T$ is said to be enabled at time $k$ if $M_{k}\left(p_{i}\right) \geq$ $W\left(p_{i}, t_{j}\right)$ for all $p_{i} \in P$ such that $\left(p_{i}, t_{j}\right) \in F$. It is assumed that at each time $k$ there exists at least one transition to fire. If a transition is enabled then, it can fire. If an enabled transition $t_{j} \in T$ fires at time $k$ then, the next marking for $p_{i} \in P$ is given by

$$
M_{k+1}\left(p_{i}\right)=M_{k}\left(p_{i}\right)+W\left(t_{j}, p_{i}\right)-W\left(p_{i}, t_{j}\right) .
$$

Let $A=\left[a_{i j}\right]$ denote an $n \times m$ matrix of integers (the incidence matrix) where $a_{i j}=a_{i j}^{+}-a_{i j}^{-}$with $a_{i j}^{+}=W\left(t_{i}, p_{j}\right)$ and $a_{i j}^{-}=W\left(p_{j}, t_{i}\right)$. Let $u_{k} \in\{0,1\}^{n}$ denote a firing vector where if $t_{j} \in T$ is fired then, its corresponding firing vector is $u_{k}=[0, \ldots, 0,1,0, \ldots, 0]^{T}$ with the one in the $j^{\text {th }}$ position in the vector and zeros everywhere else. The matrix equation (nonlinear difference equation) describing the dynamical behavior represented by a $P N$ is:

$$
M_{k+1}=M_{k}+A^{T} u_{k}
$$

where if at step $k, a_{i j}^{-}<M_{k}\left(p_{j}\right)$ for all $p_{i} \in P$ then, $t_{i} \in T$ is enabled and if this $t_{i} \in T$ fires then, its corresponding firing vector $u_{k}$ is utilized in the difference equation to generate the next step. Notice that if $M^{\prime}$ can be reached 
from some other marking $M$ and, if we fire some sequence of $d$ transitions with corresponding firing vectors $u_{0}, u_{1}, \ldots, u_{d-1}$ we obtain that

$$
M^{\prime}=M+A^{T} u, u=\sum_{k=0}^{d-1} u_{k} .
$$

Let $\left(N_{n_{0}}^{m}, d\right)$ be a metric space where $d: N_{n_{0}}^{m} \times N_{n_{0}}^{m} \rightarrow R_{+}$is defined by

$$
d\left(M_{1}, M_{2}\right)=\sum_{i=1}^{m} \zeta_{i}\left|M_{1}\left(p_{i}\right)-M_{2}\left(p_{i}\right)\right| ; \zeta_{i}>0
$$

and consider the matrix difference equation which describes the dynamical behavior of the discrete event system modeled by a $P N$

$$
M^{\prime}=M+A^{T} u, u=\sum_{k=0}^{d-1} u_{k}
$$

where, $M \in N^{m}$, denotes the marking (state) of the $P N, A \in Z^{n \times m}$, its incidence matrix and $u \in N^{n}$, is a sequence of firing vectors. Then, the following results concerns in what to the stability problem means.

Proposition 9. Let $P N$ be a Petri net. $P N$ is uniform practical stable if there exists a $\Phi$ strictly positive $m$ vector such that

$$
\Delta v=u^{T} A \Phi \leq 0
$$

Moreover, $P N$ is uniform practical asymptotic stable if the following equation holds

$$
\Delta v=u^{T} A \Phi \leq-c(e), \text { for } c \in \mathcal{K}
$$

Lemma 10. Let suppose that Proposition (9) holds then,

$$
\Delta v=u^{T} A \Phi \leq 0 \Leftrightarrow A \Phi \leq 0
$$

Remark 11. Notice that since the state space of a TPN is contained in the state space of the same now not timed PN, stability of PN implies stability of the TPN. 


\subsection{Lyapunov Stabilization}

Notice, that in the solution of the stability problem, the $u$ vector does not play any role, so why not to take advantage of it in order to get some specific behavior.

Consider the matrix difference equation which describes the dynamical behavior of the discrete event system modeled by a Petri net

$$
M^{\prime}=M+A^{T} u
$$

We are interested in finding a firing sequence vector, control law, such that system (7) remains bounded.

Definition 12. Let $P N$ be a Petri net. $P N$ is said to be stabilizable if there exists a firing transition sequence with transition count vector $u$ such that system (7) remains bounded.

Proposition 13. Let $P N$ be a Petri net. $P N$ is stabilizable if there exists a firing transition sequence with transition count vector $u$ such that the following equation holds

$$
\Delta v=A^{T} u \leq 0
$$

Remark 14. This result was first stated and proved in [5] and it relies in the use of vector Lyapunov functions. It is important to underline that by fixing a particular $u$, which satisfies (11), we restrict the state space to those markings (states) that are finite. The technique can be utilized to get some type of regulation and/or eliminate some undesirable events (transitions). Notice that in general $(8) \nRightarrow(11)$ and that the opposite is also true

\section{Max-Plus Algebra[6, 7]}

In this section the concept of max-plus algebra is defined. Its algebraic structure is described. Matrices and graphs are presented. The spectral theory of matrices is discussed. Finally the problem of solving linear equations is addressed.

\subsection{Basic Definitions}

Notation. $\mathbb{N}$ is the set of natural numbers, $\mathbb{R}$ is the set of real numbers, $\mathbb{R}^{+}$is the set of positive real numbers, $\epsilon=-\infty, e=0, \mathbb{R}_{\max }=\mathbb{R} \cup\{\epsilon\}$, 
$\underline{n}=1,2, \ldots, n$

Let $a, b \in \mathbb{R}_{\max }$ and define the operations $\oplus$ and $\otimes$ by:

$$
a \oplus b=\max (a, b) \text { and } a \otimes b=a+b .
$$

(Notice that: $a \oplus \epsilon=\epsilon+a=a$ and $a \otimes e=e \otimes a=a, \forall a \in \mathbb{R}_{\max }$.)

Definition 15. The set $\mathbb{R}_{\max }$ with the two operations $\oplus$ and $\otimes$ is called a max-plus algebra and is denoted by $\Re_{\max }=\left(\mathbb{R}_{\max }, \oplus, \otimes, \epsilon, e\right)$.

Definition 16. A semiring is a nonempty set $R$ endowed with two operations $\oplus_{R}, \otimes_{R}$, and two elements $\epsilon_{R}$ and $e_{R}$ such that:

- $\oplus_{R}$ is associative and commutative with zero element $\epsilon_{R}$;

- $\otimes_{R}$ is associative, distributes over $\oplus_{R}$, and has unit element $e_{R}$,

- $\epsilon_{R}$ is absorbing for $\otimes_{R}$ i.e., $a \otimes_{R} \epsilon=\epsilon_{R} \otimes a=a, \forall a \in R$.

Such a semiring is denoted by $\Re=\left(R, \oplus_{R}, \otimes_{R}, \epsilon, e\right)$. In addition if $\otimes_{R}$ is commutative then $R$ is called a commutative semiring, and if $\oplus_{R}$ is such that $a \oplus_{R} a=a, \forall a \in R$ then it is called idempotent.

Theorem 17. The max-plus algebra $\Re_{\max }=\left(\mathbb{R}_{\max }, \oplus, \otimes, \epsilon, e\right)$ has the algebraic structure of a commutative and idempotent semiring.

\subsection{Matrices and Graphs}

Let $\mathbb{R}_{\max }^{n \times n}$ be the set of $n \times n$ matrices with coefficients in $\mathbb{R}_{\max }$ with the following operations:

- The sum of matrices $A, B \in \mathbb{R}_{\max }^{n \times n}$, denoted $A \oplus B$ is defined by:

$$
(A \oplus B)_{i j}=a_{i j} \oplus b_{i j}=\max \left(a_{i j}, b_{i j}\right)
$$

for $i$ and $j \in \underline{n}$.

- The product of matrices $A \in \mathbb{R}_{\max }^{n \times l}, B \in \mathbb{R}_{\max }^{l \times n}$, denoted $A \otimes B$ is defined by:

$$
(A \oplus B)_{i k}=\bigoplus_{j=1}^{l} a_{i j} \otimes b_{j k}=\max _{j \in \underline{L}}\left\{a_{i j}+b_{j k}\right\}
$$

for $i$ and $k \in \underline{n}$. (Notice that the matrix product in general fails to be commutative.) 
- The scalar product for $\alpha \in \mathbb{R}_{\max }^{n}$ and $A \in \mathbb{R}_{\max }^{n \times n}$, denoted $\alpha \otimes A$ is defined by:

$$
(\alpha \oplus A)_{i j}=\alpha \otimes a_{i j}
$$

for $i$ and $j \in \underline{n}$.

Let $\mathcal{E} \in \mathbb{R}_{\max }^{n \times n}$ denote the matrix with all its elements equal to $\epsilon$ and denote by $E \in \mathbb{R}_{\max }^{n \times n}$ the matrix which has its diagonal elements equal to $e$ and all the other elements equal to $\epsilon$. Then, the following result, whose proof is immediate, can be stated.

Theorem 18. The 5-tuple $\Re_{\max }^{n \times n}=\left(\mathbb{R}_{\max }^{n \times n}, \oplus, \otimes, \mathcal{E}, E\right)$ has the algebraic structure of a noncommutative idempotent semiring.

Definition 19. Let $A \in \mathbb{R}_{\max }^{n \times n}$ and $k \in \mathbb{N}$ then the $k$-th power of $A$ denoted by $A^{\otimes k}$ is defined by:

$$
A^{\otimes k}=\underbrace{A \otimes A \otimes \cdots \otimes A}_{k-\text { times }}
$$

where $A^{\otimes 0}$ is set equal to $E$.

Definition 20. A matrix $A \in \mathbb{R}_{\max }^{n \times n}$ is said to be regular if $A$ contains at least one element distinct from $\epsilon$ in each row.

Next, an overview in the theory of graphs will be given, emphasizing the rich relationship that exist between them and matrices.

Definition 21. Let $\mathcal{N}$ be a finite and non-empty set and consider $\mathcal{D} \subseteq$ $\mathcal{N} \times \mathcal{N}$. The pair $G=(\mathcal{N}, \mathcal{D})$ is called a directed graph, where $\mathcal{N}$ is the set of elements called nodes and $\mathcal{D}$ is the set of ordered pairs of nodes called arcs. A directed graph $G=(\mathcal{N}, \mathcal{D})$ is called a weighted graph if a weight $w(i, j) \in \mathbb{R}$ is associated with any arc $(i, j) \in \mathcal{D}$.

Let $A \in \mathbb{R}_{\max }^{n \times n}$ be any matrix, a graph $\mathcal{G}(A)$, called the communication graph of $A$, can be associated as follows. Define $\mathcal{N}(A)=\underline{n}$ and a pair $(i, j) \in \underline{n} \times \underline{n}$ will be a member of $\mathcal{D}(A) \Leftrightarrow a_{j i} \neq \epsilon$, where $\mathcal{D}(A)$ denotes the set of arcs of $\mathcal{G}(A)$.

Definition 22. A path from node $i$ to node $j$ is a sequence of arcs $p=$ $\left\{\left(i_{k}, j_{k}\right) \in \mathcal{D}(A)\right\}_{k \in m}$ such that $i=i_{1}, j_{k}=i_{k+1}$, for $k<m$ and $j_{m}=j$. The path $p$ consists of the nodes $i=i_{1}, i_{2}, \ldots, i_{m}, j_{m}=j$ with length $m$ denoted by $|p|_{1}=m$. In the case when $i=j$ the path is said to be a circuit. A circuit is said to be elementary if nodes $i_{k}$ and $i_{l}$ are different for $k \neq l$. A circuit consisting of one arc is called a self-loop. 
Let us denote by $P(i, j ; m)$ the set of all paths from node $i$ to node $j$ of length $m \geq 1$ and for any arc $(i, j) \in \mathcal{D}(A)$ let its weight be given by $a_{i j}$ then the weight of a path $p \in P(i, j ; m)$ denoted by $|p|_{w}$ is defined to be the sum of the weights of all the arcs that belong to the path. The average weight of a path $p$ is given by $|p|_{w} /|p|_{1}$. Given two paths, as for example, $p=\left(\left(i_{1}, i_{2}\right),\left(i_{2}, i_{3}\right)\right)$ and $q=\left(\left(i_{3}, i_{4}\right),\left(\left(i_{4}, i_{5}\right)\right.\right.$ in $\mathcal{G}(A)$ the concatenation of paths $\circ: \mathcal{G}(A) \times \mathcal{G}(A) \rightarrow \mathcal{G}(A)$ is defined as $p \circ q=\left(\left(i_{1}, i_{2}\right),\left(i_{2}, i_{3}\right),\left(i_{3}, i_{4}\right),\left(i_{4}, i_{5}\right)\right)$.

The communication graph $\mathcal{G}(A)$ and powers of matrix $A$ are closely related as it is shown in the next theorem, whose proof follows using induction on the length $k$ of the path (see [1]).

Theorem 23. Let $A \in \mathbb{R}_{\max }^{n \times n}$, then $\forall k \geq 1$ :

$$
\left[A^{\otimes k}\right]_{j i}=\max \left\{|p|_{w}: p \in P(i, j ; k)\right\}
$$

where $\left[A^{\otimes k}\right]_{j i}=\epsilon$ in the case when $P(i, j ; k)$ is empty i.e., no path of length $k$ from node $i$ to node $j$ exists in $\mathcal{G}(A)$.

Definition 24. Let $A \in \mathbb{R}_{\max }^{n \times n}$ then define the matrix $A^{+} \in \mathbb{R}_{\max }^{n \times n}$ as:

$$
A^{+}=\bigoplus_{k=1}^{\infty} A^{\otimes k}
$$

sometimes known as the shortest path matrix. Where the element $\left[A^{+}\right]_{j i}$ gives the maximal weight of any path from $j$ to $i$. If in addition one wants to add the possibility of staying at a node then one must include matrix $E$ in the definition of matrix $A^{+}$giving rise to its Kleene star representation defined by:

$$
A^{*}=\bigoplus_{k=0}^{\infty} A^{\otimes k} .
$$

Lemma 25. Let $A \in \mathbb{R}_{\max }^{n \times n}$ be such that any circuit in $\mathcal{G}(A)$ has average circuit weight less than or equal to $\epsilon$. Then it holds that:

$$
A^{*}=\bigoplus_{k=0}^{n-1} A^{\otimes k} .
$$

Definition 26. Let $G=(\mathcal{N}, \mathcal{D})$ be a graph and $i, j \in \mathcal{N}$, node $j$ is reachable from node $i$, denoted as $i \mathcal{R} j$, if there exists a path from $i$ to $j$. A graph $G$ is said to be strongly connected if $\forall i, j \in \mathcal{N}, j \mathcal{R} i$. A matrix $A \in \mathbb{R}_{\max }^{n \times n}$ is called irreducible if its communication graph is strongly connected, when this is not the case matrix $A$ is called reducible. 
Definition 27. Let $G=(\mathcal{N}, \mathcal{D})$ be a not strongly connected graph and $i, j \in \mathcal{N}$, node $j$ communicates with node $i$, denoted as $i \mathcal{C} j$, if either $i=j$ or $i \mathcal{R} j$ and $j \mathcal{R} i$.

The relation $i \mathcal{C} j$ defines an equivalence relation in the set of nodes, and therefore a partition of $\mathcal{N}$ into a disjoint union of subsets, the equivalence classes, $\mathcal{N}_{1}, \mathcal{N}_{2}, \ldots, \mathcal{N}_{q}$ such that $\mathcal{N}=\mathcal{N}_{1} \cup \mathcal{N}_{2} \cup \ldots \cup \mathcal{N}_{q}$ or $\mathcal{N}=\bigcup_{i \in \mathcal{N}}[i] ;[i]=$ $\{j \in \mathcal{N}: i C j\}$.

Given the above partition, it is possible to focus on subgraphs of $G$ denoted by $G_{r}=\left(\mathcal{N}_{r}, \mathcal{D}_{r}\right) ; r \in \underline{q}$ where $\mathcal{D}_{r}$ denotes the subset of arcs, which belong to $\mathcal{D}$, that have both the begin node and end node in $\mathcal{N}_{r}$. If $\mathcal{D}_{r} \neq \varnothing$, the subgraph $G_{r}=\left(\mathcal{N}_{r}, \mathcal{D}_{r}\right)$ is known as a maximal strongly connected subgraph of $G$.

Remark 28. In case of having an isolated node $i$ (i.e., a node that does not communicate with any other node) and which does not even have an arc from it to itself, the associated subgraph is given by $([i], \varnothing)$ which is not strongly connected however, for convenience it will be considered as if it were.

Definition 29. The reduced graph $\widetilde{G}=(\widetilde{\mathcal{N}}, \widetilde{\mathcal{D}})$ of $G$ is defined by setting $\widetilde{\mathcal{N}}=\left\{\left[i_{1}\right],\left[i_{2}\right], \ldots\left[i_{q}\right]\right\}$ and $\left(\left[i_{r}\right],\left[i_{s}\right]\right) \in \widetilde{\mathcal{D}}$ if $r \neq s$ and there exists an arc $(k, l) \in \mathcal{D}$ for some $k \in\left[i_{r}\right]$ and $l \in\left[i_{s}\right]$.

Let $A_{r r}$ denote the matrix by restricting $A$ to the nodes in $\left[i_{r}\right] \forall r \in \underline{q}$ i.e., $\left[A_{r r}\right]_{k l}=a_{k l} \forall k, l \in\left[i_{r}\right]$. Then $\forall r \in \underline{q}$ either $A_{r r}$ is irreducible or is equal to $\epsilon$. Therefore since by construction the reduced graph does not contain any circuits, the original reducible matrix $A$ after a possible relabeling of the nodes in $G(A)$, can be written as:

$$
A=\left(\begin{array}{ccccc}
A_{11} & A_{12} & \cdots & \cdots & A_{1 q} \\
\mathcal{E} & A_{22} & \cdots & \cdots & A_{2 q} \\
\mathcal{E} & \mathcal{E} & A_{33} & & \vdots \\
\vdots & \vdots & \ddots & \ddots & \vdots \\
\mathcal{E} & \mathcal{E} & \cdots & \mathcal{E} & A_{q q}
\end{array}\right)
$$

with matrices $A_{s r} 1 \leq s<r \leq q$ of suitable size, where each finite entry in $A_{s r}$ corresponds to an arc from a node in $\left[i_{r}\right]$ to a node in $\left[i_{s}\right]$.

Definition 30. Let $A \in \mathbb{R}_{\max }^{n \times n}$ be a reducible matrix then, the block upper triangular given by (21) is said to be a normal form of matrix $A$. 


\subsubsection{Spectral Theory}

Definition 31. Let $A \in \mathbb{R}_{\max }^{n \times n}$ be a matrix. If $\mu \in R_{\max }$ is a scalar and $v \in R_{\max }^{n}$ is a vector that contains at least one finite element such that:

$$
A \otimes v=\mu \otimes v
$$

then, $\mu$ is called an eigenvalue and $v$ an eigenvector.

Remark 32. Notice that the eigenvalue can be equal to $\epsilon$ and is not necessarily unique. Eigenvectors are certainly not unique indeed, if $v$ is an eigenvector then $\alpha \otimes v$ is also an eigenvector for all $\alpha \in \mathbb{R}$.

Let $\mathcal{C}(A)$ denote the set of all elementary circuits in $\mathcal{G}(A)$ and write:

$$
\lambda=\max _{p \in \mathcal{C}(A)} \frac{|p|_{w}}{|p|_{1}}
$$

for the maximal average circuit weight. Notice that since $\mathcal{C}(A)$ is a finite set, the maximum of (23) is attained (which is always the case when matrix $A$ is irreducible). In case $\mathcal{C}(A)=\emptyset$ define $\lambda=\epsilon$.

Definition 33. A circuit $p \in G(A)$ is said to be critical if its average weight is maximal. The critical graph of $A$, denoted by $G^{c}(A)=\left(\mathcal{N}^{c}(A), \mathcal{D}^{c}(A)\right)$, is the graph consisting of those nodes and arcs that belong to critical circuits in $G(A)$.

Lemma 34. Let assume that $G(A)$ contains at least one circuit then, any circuit in $G^{c}(A)$ is critical.

Definition 35. Let $A \in \mathbb{R}_{\max }^{n \times n}$ be a matrix and $\mu$ an eigenvalue of $A$ with associated eigenvector $v$ then, the support of $v$ consists of the set of nodes of $G(A)$ which correspond to finite entries of $v$.

Lemma 36. Let $A \in \mathbb{R}_{\max }^{n \times n}$ be an irreducible matrix then any $v \in R_{\max }^{n}$ which satisfies (22) has all components different from $\epsilon$.

Next, the most important result of this sub-section is given.

Theorem 37. If $A \in \mathbb{R}_{\max }^{n \times n}$ is irreducible, then there exists one and only one finite eigenvalue (with possible several eigenvectors). This eigenvalue is equal to the maximal average weight of circuits in $G(A)$ :

$$
\lambda(A)=\max _{p \in \mathcal{C}(A)} \frac{|p|_{w}}{|p|_{1}}
$$




\subsubsection{Linear Equations}

Theorem 38. Let $A \in \mathbb{R}_{\max }^{n \times n}$ and $b \in \mathbb{R}_{\max }^{n}$. If the communication graph $G(A)$ has maximal average circuit weight less than or equal to $e$, then $x=A^{*} \otimes b$ solves the equation $x=(A \otimes x) \oplus b$. Moreover, if the circuit weights in $G(a)$ are negative then, the solution is unique.

\section{Max-Plus Recurrence Equations for Timed Event Petri Nets}

Definition 39. Let $A_{m} \in \mathbb{R}_{\max }^{n \times n}$ for $0 \leq m \leq M$ and $x(m) \in \mathbb{R}_{\max }^{n}$ for $-M \leq m \leq-1 ; M \geq 0$. Then, the recurrence equation:

$$
x(k)=\bigoplus_{m=0}^{M} A_{m} \otimes x(k-m) ; k \geq 0
$$

is called an Mth order recurrence equation.

Theorem 40. The $M$ th order recurrence equation, given by equation (25), can be transformed into a first order recurrence equation $x(k+1)=A \otimes x(k)$; $k \geq 0$ provided that $A_{0}$ has circuit weights less than or equal to zero.

With any timed event Petri net, matrices $A_{0}, A_{1}, \ldots, A_{M} \in \mathbb{N}^{n} \times \mathbb{N}^{n}$ can be defined by setting $\left[A_{m}\right]_{j l}=a_{j l}$, where $a_{j l}$ is the largest of the holding times with respect to all places between transitions $t_{l}$ and $t_{j}$ with $m$ tokens, for $m=0,1, \ldots, M$, with $M$ equal to the maximum number of tokens with respect to all places. Let $x_{i}(k)$ denote the $k$ th time that transition $t_{i}$ fires, then the vector $x(k)=\left(x_{1}(k), x_{2}(k), \ldots x_{m}(k)\right)^{T}$, called the state of the system, satisfies the $M$ th order recurrence equation:

$$
x(k)=\bigoplus_{m=0}^{M} A_{m} \otimes x(k-m) ; k \geq 0
$$

Now, assuming that all the hypothesis of theorem (40) are satisfied, and setting $\hat{x}(k)=\left(x^{T}(k), x^{T}(k-1), \ldots, x^{T}(k-M+1)\right)^{T}$, equation (26) can be expressed as:

$$
\hat{x}(k+1)=\hat{A} \otimes \hat{x}(k) ; k \geq 0
$$

which is known as the standard autonomous equation. 


\section{The Solution to the Stability Problem for Queuing Systems Modeled with timed Petri Nets}

This section defines what it means for a $T P N$ to be stable, then gathering the results previously presented in the past sections the solution to the problem is obtained.

Definition 41. A TPN is said to be stable if all the transitions fire with the same proportion i.e., if there exists $q \in \mathbb{N}$ such that

$$
\lim _{k \rightarrow \infty} \frac{x_{i}(k)}{k}=q, \forall i=1, \ldots, n
$$

This last definition tell us that in order to obtain a stable TPN all the transitions have to be fired $q$ times. However, it will be desirable to be more precise and know exactly how many times. The answer to this question is given next.

Lemma 42. Consider the recurrence relation $x(k+1)=A \otimes x(k), k \geq 0$, $x(0)=x_{0} \in \mathbb{R}^{n}$ arbitrary. $A$ an irreducible matrix and $\lambda \in \mathbb{R}$ its eigenvalue then,

$$
\lim _{k \rightarrow \infty} \frac{x_{i}(k)}{k}=\lambda, \forall i=1, \ldots, n .
$$

Now starting with an unstable TPN, collecting the results given by: proposition (13), what has just been discussed about recurrence equations for TPN at the end of Section 4 and the previous lemma (42) plus theorem (37), the solution to the problem is obtained.

\section{Modeling and Stability Analysis for a Type of Queuing Systems}

In this section the modeling, stability analysis and scheduling design for queuing systems is addressed. Two classes of queues are considered. Two classes of queues are considered. The first one (one and two servers) working under normal operation, while the second one (one server) having failures in its normal operation due to server breakdown and consumer interruption during service.

\section{Case I:Normal operation}

\section{Case Ia: One server}

Consider a simple one server queuing system (Fig 1.) whose TPN model is depicted in Fig 2. Where the events (transitions) that drive the system are: q: customers arrive to the queue, s: service starts, d: the customer departs. 


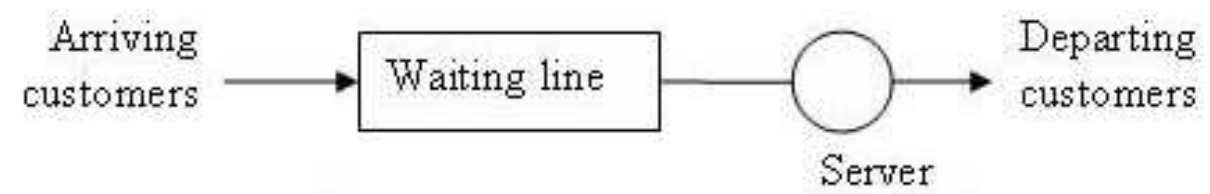

Figure 1: One server queuing system

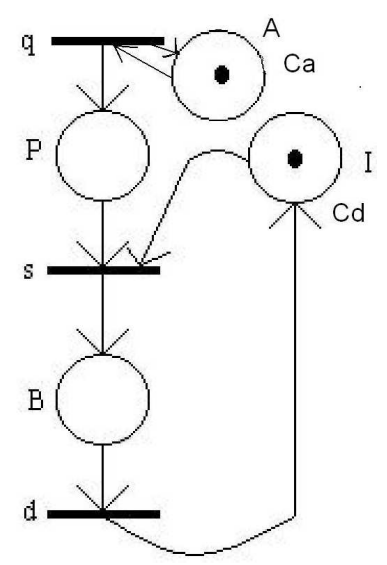

Figure 2: Timed Petri net model

The places (that represent the states of the queue) are: A: customers arriving, $\mathrm{P}$ : the customers are waiting for service in the queue, B: the customer is being served, I: the server is idle. The holding times associated to the places $\mathrm{A}$ and I are $C a$ and $C d$ respectively, (with $C a>C d$ ). The incidence matrix that represents the $P N$ model is

$$
A=\left[\begin{array}{cccc}
0 & 1 & 0 & 0 \\
0 & -1 & 1 & -1 \\
0 & 0 & -1 & 1
\end{array}\right]
$$

Therefore since there does not exists a $\Phi$ strictly positive $m$ vector such that $A \Phi \leq 0$ the sufficient condition for stability is not satisfied. Moreover, the $P N(T P N)$ is unbounded since by the repeated firing of $\mathrm{q}$, the marking in $\mathrm{P}$ grows indefinitely. However, by taking $u=[k, k, k] ; k>0$ (but unknown), we get that $A^{T} u \leq 0$. Therefore, the $P N$ is stabilizable which implies that the $T P N$ is stable. Now, let us proceed to determine the exact value of $k$. From 


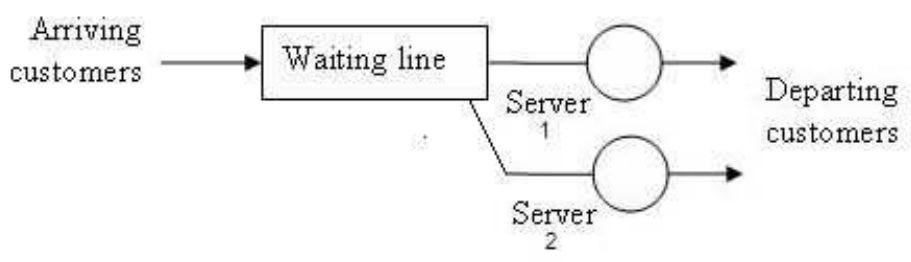

Figure 3: Two server queuing system

the $T P N$ model we obtain that:

$$
A_{0}=\left(\begin{array}{lll}
\varepsilon & \varepsilon & \varepsilon \\
0 & \varepsilon & \varepsilon \\
\varepsilon & 0 & \varepsilon
\end{array}\right)
$$

and

$$
A_{1}=\left(\begin{array}{ccc}
C a & \varepsilon & \varepsilon \\
\varepsilon & \varepsilon & C d \\
\varepsilon & \varepsilon & \varepsilon
\end{array}\right) .
$$

and making the required computations that:

$$
A_{0}^{*}=\left(\begin{array}{ccc}
0 & \varepsilon & \varepsilon \\
0 & 0 & \varepsilon \\
0 & 0 & 0
\end{array}\right),
$$

leading to:

$$
\hat{A}=A_{0}^{*} \otimes A_{1}=\left(\begin{array}{ccc}
C a & \varepsilon & \varepsilon \\
C a & \varepsilon & C d \\
C a & \varepsilon & C d
\end{array}\right)
$$

Therefore, $\lambda(A)=\max _{p \in \mathcal{C}(A)} \frac{|p|_{\mathrm{w}}}{|p|_{1}}=\max \{C a, C d\}=C a$. This means that in order for the $T P N$ to be stable and work properly the speed at which the one server queuing system works has to be equal to $\mathrm{Ca}$ or being more precise, that all the transitions must fire at the same speed as the customers arrive i.e., they have to be served as soon as they arrive to the queue which is attained by setting $k=C a$.

\section{Case Ib: Two Server}

Consider a two server queuing system (Fig 3.) whose TPN model is depicted in Fig 4. Where the events (transitions) that drive the system are: q: customers arrive to the queue, s1, s2: service starts, d1,d2: the customer departs. The places (that represent the states of the queue) are: A: customers 


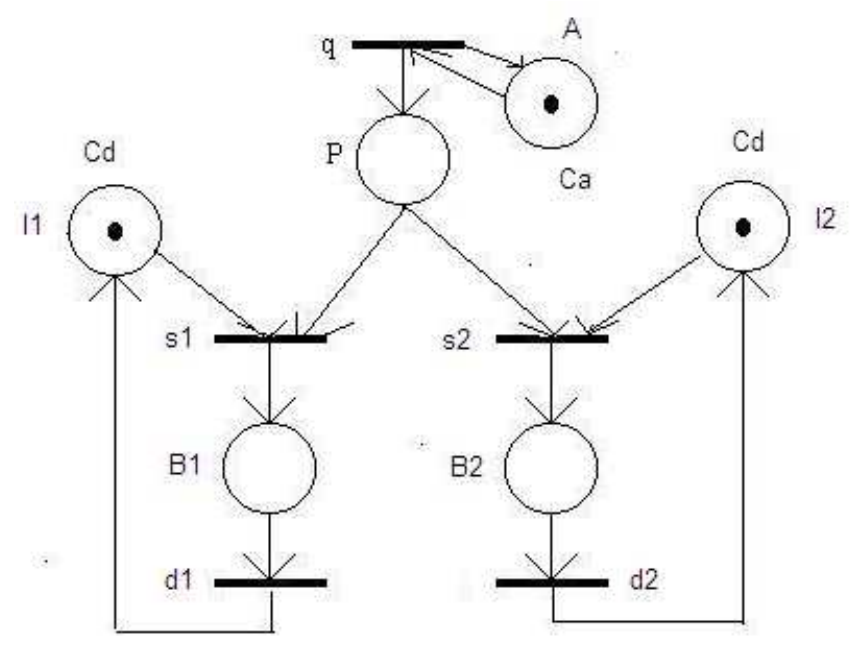

Figure 4: Timed Petri net model

arriving, P: the customers are waiting for service in the queue, B1, B2: the customer is being served, I1, I2: the servers are idle. The holding times associated to the places $\mathrm{A}$ and I1, I2 are $C a$ and $C d$ respectively, (with $C a>C d$ ). The incidence matrix that represents the $P N$ model is

$$
A=\left[\begin{array}{cccccc}
0 & 1 & 0 & 0 & 0 & 0 \\
0 & -1 & 1 & -1 & 0 & 0 \\
0 & -1 & 0 & 0 & 1 & -1 \\
0 & 0 & -1 & 1 & 0 & 0 \\
0 & 0 & 0 & 0 & -1 & 1
\end{array}\right]
$$

Therefore since there does not exists a $\Phi$ strictly positive $m$ vector such that $A \Phi \leq 0$ the sufficient condition for stability is not satisfied. Moreover, the $P N(T P N)$ is unbounded since by the repeated firing of q, the marking in $\mathrm{P}$ grows indefinitely. However, by taking $u=[k, k / 2, k / 2, k / 2, k / 2] ; k>0$ (but unknown) we get that $A^{T} u \leq 0$. Therefore, the $P N$ is stabilizable which implies that the TPN is stable. Now, let us proceed to determine the exact value of $k$. From the TPN model we obtain that:

$$
A_{0}=\left(\begin{array}{lllll}
\varepsilon & \varepsilon & \varepsilon & \varepsilon & \varepsilon \\
0 & \varepsilon & \varepsilon & \varepsilon & \varepsilon \\
0 & \varepsilon & \varepsilon & \varepsilon & \varepsilon \\
\varepsilon & 0 & \varepsilon & \varepsilon & \varepsilon \\
\varepsilon & \varepsilon & 0 & \varepsilon & \varepsilon
\end{array}\right)
$$


and

$$
A_{1}=\left(\begin{array}{ccccc}
C a & \varepsilon & \varepsilon & \varepsilon & \varepsilon \\
\varepsilon & \varepsilon & \varepsilon & C d & \varepsilon \\
\varepsilon & \varepsilon & \varepsilon & \varepsilon & C d \\
\varepsilon & \varepsilon & \varepsilon & \varepsilon & \varepsilon \\
\varepsilon & \varepsilon & \varepsilon & \varepsilon & \varepsilon
\end{array}\right)
$$

and making the required computations that:

$$
A_{0}^{*}=\left(\begin{array}{ccccc}
0 & \varepsilon & \varepsilon & \varepsilon & \varepsilon \\
0 & 0 & \varepsilon & \varepsilon & \varepsilon \\
0 & \varepsilon & 0 & \varepsilon & \varepsilon \\
0 & 0 & \varepsilon & 0 & \varepsilon \\
0 & \varepsilon & 0 & \varepsilon & 0
\end{array}\right)
$$

leading to:

$$
\hat{A}=A_{0}^{*} \otimes A_{1}=\left(\begin{array}{ccccc}
C a & \varepsilon & \varepsilon & \varepsilon & \varepsilon \\
C a & \varepsilon & \varepsilon & C d & \varepsilon \\
C a & \varepsilon & \varepsilon & \varepsilon & C d \\
C a & 0 & \varepsilon & C d & \varepsilon \\
C a & \varepsilon & \varepsilon & \varepsilon & C d
\end{array}\right)
$$

Therefore, $\lambda(A)=\max _{p \in \mathcal{C}(A)} \frac{|p|_{\mathrm{w}}}{|p|_{1}}=\max \{C a, C d\}=C a$. This means that in order for the TPN to be stable and work properly the speed at which the two sever queuing system works has to be equal to $C a$ which is attained by taking $k=C a$, i.e., the load has to be equally divided between the two servers.

Remark 43. This case is easily extended to the case with $n$ servers, obtaining that $u=[\mathrm{Ca}, \mathrm{Ca} / \mathrm{n}, \mathrm{Ca} / \mathrm{n}, \ldots, \mathrm{Ca} / \mathrm{n}]$.

Next, queueing systems with failures are considered.

\section{Case II: Fault queueing systems}

\section{Case IIa: Server breakdown}

Consider a simple one server queuing system whose regular operation fails due to server breakdown. Its $T P N$ model is depicted in Fig 5, where the events (transitions) that drive the system are: q: consumers arrive to the queue, s: service starts, b: the server breaks, $r$ : the service is restored, d: the customer departs. The places (that represent the states of the queue) are: A: consumers arriving, P: the consumers are waiting for service in the queue, B: the consumer is being served, D: service out of order, I: the server is idle. The holding times 


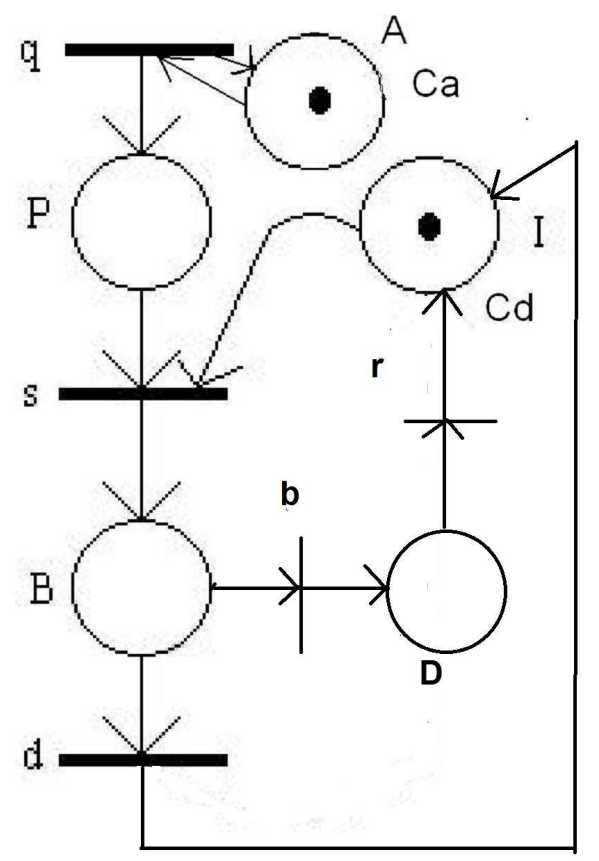

Figure 5: Server breakdown timed Petri net model

associated to the places A and I are $C a$ and $C d$ respectively, (with $C a>C d$ ). The incidence matrix that represents the $P N$ model is

$$
A=\left[\begin{array}{ccccc}
0 & 1 & 0 & 0 & 0 \\
0 & -1 & 1 & -1 & 0 \\
0 & 0 & -1 & 1 & 0 \\
0 & 0 & -1 & 0 & 1 \\
0 & 0 & 0 & 1 & -1
\end{array}\right]
$$

Therefore since there does not exists a $\Phi$ strictly positive $m$ vector such that $A \Phi \leq 0$ the sufficient condition for stability is not satisfied, (moreover, the $P N$ $(T P N)$ is unbounded since by the repeated firing of $\mathrm{q}$, the marking in $\mathrm{P}$ grows indefinitely). However, by taking $u=[k, k, k / 2, k / 2, k / 2] ; k>0$ (but unknown) we get that $A^{T} u \leq 0$. Therefore, the $P N$ is stabilizable which implies that the $T P N$ is stable. Now, let us proceed to determine the exact value of $k$. From 
the TPN model we obtain that:

$$
A_{0}=\left(\begin{array}{lllll}
\varepsilon & \varepsilon & \varepsilon & \varepsilon & \varepsilon \\
0 & \varepsilon & \varepsilon & \varepsilon & \varepsilon \\
\varepsilon & 0 & \varepsilon & \varepsilon & \varepsilon \\
\varepsilon & \varepsilon & \varepsilon & \varepsilon & \varepsilon \\
\varepsilon & \varepsilon & \varepsilon & 0 & \varepsilon
\end{array}\right)
$$

and

$$
A_{1}=\left(\begin{array}{ccccc}
C a & \varepsilon & \varepsilon & \varepsilon & \varepsilon \\
\varepsilon & \varepsilon & C d & \varepsilon & C d \\
\varepsilon & \varepsilon & \varepsilon & \varepsilon & \varepsilon \\
\varepsilon & \varepsilon & \varepsilon & \varepsilon & \varepsilon \\
\varepsilon & \varepsilon & \varepsilon & \varepsilon & \varepsilon
\end{array}\right)
$$

which implies

$$
A_{0}^{*}=\left(\begin{array}{ccccc}
0 & \varepsilon & \varepsilon & \varepsilon & \varepsilon \\
0 & 0 & \varepsilon & \varepsilon & \varepsilon \\
0 & 0 & 0 & \varepsilon & \varepsilon \\
\varepsilon & \varepsilon & \varepsilon & 0 & \varepsilon \\
\varepsilon & \varepsilon & \varepsilon & 0 & 0
\end{array}\right)
$$

leading to:

$$
\hat{A}=A_{0}^{*} \otimes A_{1}=\left(\begin{array}{ccccc}
C a & \varepsilon & \varepsilon & \varepsilon & \varepsilon \\
C a & \varepsilon & C d & \varepsilon & C d \\
C a & \varepsilon & C d & \varepsilon & C d \\
\varepsilon & \varepsilon & \varepsilon & \varepsilon & \varepsilon \\
\varepsilon & \varepsilon & \varepsilon & \varepsilon & \varepsilon
\end{array}\right)
$$

Therefore, $\lambda(A)=\max _{p \in \mathcal{C}(A)} \frac{|p|_{\mathrm{w}}}{|p|_{1}}=\max \{C a, C d\}=C a$. This means that in order for the TPN to be stable and work properly the speed at which the service operates has to be equal to $C a$ (the firing speed of transition q) which is attained by taking $k=C a$.

\section{Case IIb: Consumer induced interruption}

Consider a simple one server queuing system whose regular operation fails due to consumer induced interruption. Its $T P N$ model is depicted in Fig 6, where the events (transitions) that drive the system are: q: consumers arrive to the queue, s: service starts, c: the consumer interrupts the service, r: the consumer returns for service, d: the consumer departs. The places (that represent the states of the queue) are: A: consumers arriving, P: the consumers are waiting for service in the queue, B: the consumer is being served, D: the 


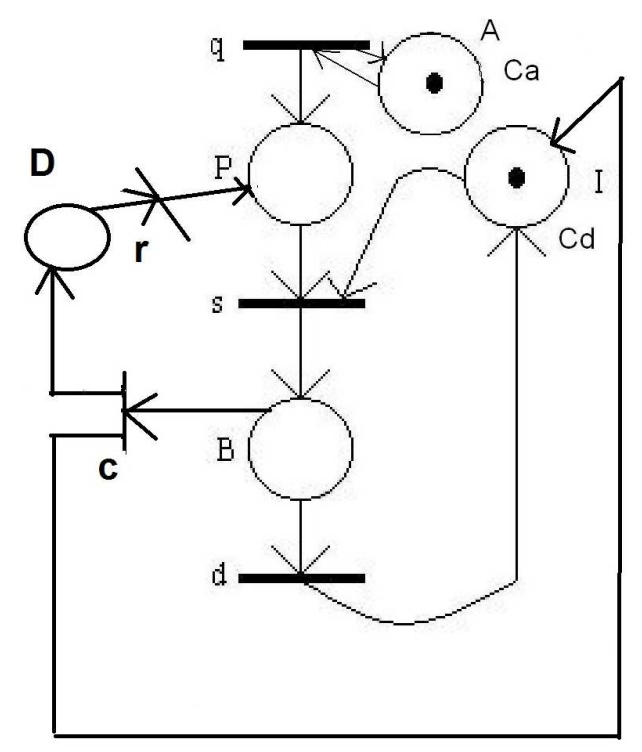

Figure 6: Consumer induced interruption timed Petri net model

consumer is busy, I: the server is idle. The holding times associated to the places $\mathrm{A}$ and I are $C a$ and $C d$ respectively, (with $C a>C d$ ). The incidence matrix that represents the $P N$ model is

$$
A=\left[\begin{array}{ccccc}
0 & 1 & 0 & 0 & 0 \\
0 & -1 & 1 & -1 & 0 \\
0 & 0 & -1 & 1 & 0 \\
0 & 0 & -1 & 1 & 1 \\
0 & 1 & 0 & 0 & -1
\end{array}\right]
$$

Therefore since there does not exists a $\Phi$ strictly positive $m$ vector such that $A \Phi \leq 0$ the sufficient condition for stability is not satisfied, (moreover, the $P N(T P N)$ is unbounded since by the repeated firing of $\mathrm{q}$, the marking in $\mathrm{P}$ grows indefinitely). However, by taking $u=[k / 2, k, k / 2, k / 2, k / 2] ; k>0$ (but unknown) we get that $A^{T} u \leq 0$. Therefore, the $P N$ is stabilizable which implies that the TPN is stable. Now, let us proceed to determine the exact value of $k$. From the TPN model we obtain that: 


$$
A_{0}=\left(\begin{array}{lllll}
\varepsilon & \varepsilon & \varepsilon & \varepsilon & \varepsilon \\
\varepsilon & 0 & \varepsilon & \varepsilon & \varepsilon \\
\varepsilon & 0 & \varepsilon & \varepsilon & \varepsilon \\
\varepsilon & \varepsilon & \varepsilon & \varepsilon & \varepsilon \\
\varepsilon & \varepsilon & \varepsilon & 0 & \varepsilon
\end{array}\right)
$$

and

$$
A_{1}=\left(\begin{array}{ccccc}
C a & \varepsilon & \varepsilon & \varepsilon & \varepsilon \\
\varepsilon & \varepsilon & C d & C d & \varepsilon \\
\varepsilon & \varepsilon & \varepsilon & \varepsilon & \varepsilon \\
\varepsilon & \varepsilon & \varepsilon & \varepsilon & \varepsilon \\
\varepsilon & \varepsilon & \varepsilon & \varepsilon & \varepsilon
\end{array}\right)
$$

which implies

$$
A_{0}^{*}=\left(\begin{array}{ccccc}
0 & \varepsilon & \varepsilon & \varepsilon & \varepsilon \\
\varepsilon & 0 & \varepsilon & \varepsilon & \varepsilon \\
\varepsilon & 0 & 0 & \varepsilon & \varepsilon \\
\varepsilon & \varepsilon & \varepsilon & 0 & \varepsilon \\
\varepsilon & \varepsilon & \varepsilon & \varepsilon & 0
\end{array}\right)
$$

leading to:

$$
\hat{A}=A_{0}^{*} \otimes A_{1}=\left(\begin{array}{ccccc}
C a & \varepsilon & \varepsilon & \varepsilon & \varepsilon \\
\varepsilon & \varepsilon & C d & C d & \varepsilon \\
\varepsilon & \varepsilon & C d & C d & \varepsilon \\
\varepsilon & \varepsilon & \varepsilon & \varepsilon & \varepsilon \\
\varepsilon & \varepsilon & \varepsilon & \varepsilon & \varepsilon
\end{array}\right)
$$

Therefore, $\lambda(A)=\max _{p \in \mathcal{C}(A)} \frac{|p|_{\mathrm{w}}}{|p|_{1}}=\max \{C a, C d\}=C a$. This means that in order for the TPN to be stable and work properly the speed at which the service operates has to be equal to $C a$ (the sum of the firing speeds of transition $q$ and r) which is attained by taking $k=C a$.

\section{Conclusions}

The main contribution of this paper consists in combining Lyapunov theory with max-plus algebra to give a complete and precise solution to the stability problem for a type of queuing systems modeled with timed Petri nets. Two classes of queues were considered. The first one (one and two servers) working under normal operation, while the second one (one server) having failures in its normal operation due to server breakdown and consumer interruption during 
service. The presented methodology applied to queuing systems results to be innovative in contrast to the classical stochastic approach usually used.

\section{References}

[1] T. Murata, Petri nets: Properties, analysis, and applications, Proc. IEEE, 77, No. 4 (1989).

[2] Z. Retchkiman, Stability theory for a class of dynamical systems modeled with Petri nets, International Journal of Hybrid Systems, 4, No. 1 (2005).

[3] S. Borst, M. Joncheere, L. Leskela, Stability of Parallel Queuing Systems with Coupled Service Rates, Report Probability, Networks and AlgorithmsE0613 (2006).

[4] V. Lakshmikantham, V.M. Matrosov, S. Sivasundaram, Vector Lyapunov Functions and Stability Analysis of Nonlinear Systems, Kluwer Academic Publ., Dordrecht (1991).

[5] Z. Retchkiman, From stability to the Stabilization problem of discrete event systems modeled by Petri nets, In: American Control Conference'99, San Diego, Cal, June (1999).

[6] B. Heidergott, G.J. Olsder, J. van der Woude, Max Plus at Work, Princeton University Press (2006).

[7] F. Baccelli, G. Cohen, G.J. Olsder, J.P. Quadrat, Synchronization and Linearity, Web-Edition (2001). 
